Umfrageergebnisse zur Digitalisierung in der Versorgung

\title{
Ärzte wollen bei Digital Healthcare Taktgeber werden
}

\begin{abstract}
Obwohl sie beim Einsatz digitaler Anwendungen noch zögerlich sind, schreiben Ärzte der Digitalisierung einen hohen Nutzen für die Versorgung zu. Das zeigt eine aktuelle Umfrage. Hemmnisse sind bislang aber oft die Sorge um die Datensicherheit und fehlende finanzielle Mittel.
\end{abstract}

Die Ärzteschaft befindet sich im digitalen Umbruch: Während $47 \%$ ihren Schriftverkehr wie beispielsweise den Arztbrief noch auf Papier erledigen und knapp über ein Drittel sogar auf die klassische analoge Patientenakte setzt, sehen $69 \%$ die Digitalisierung als Chance für

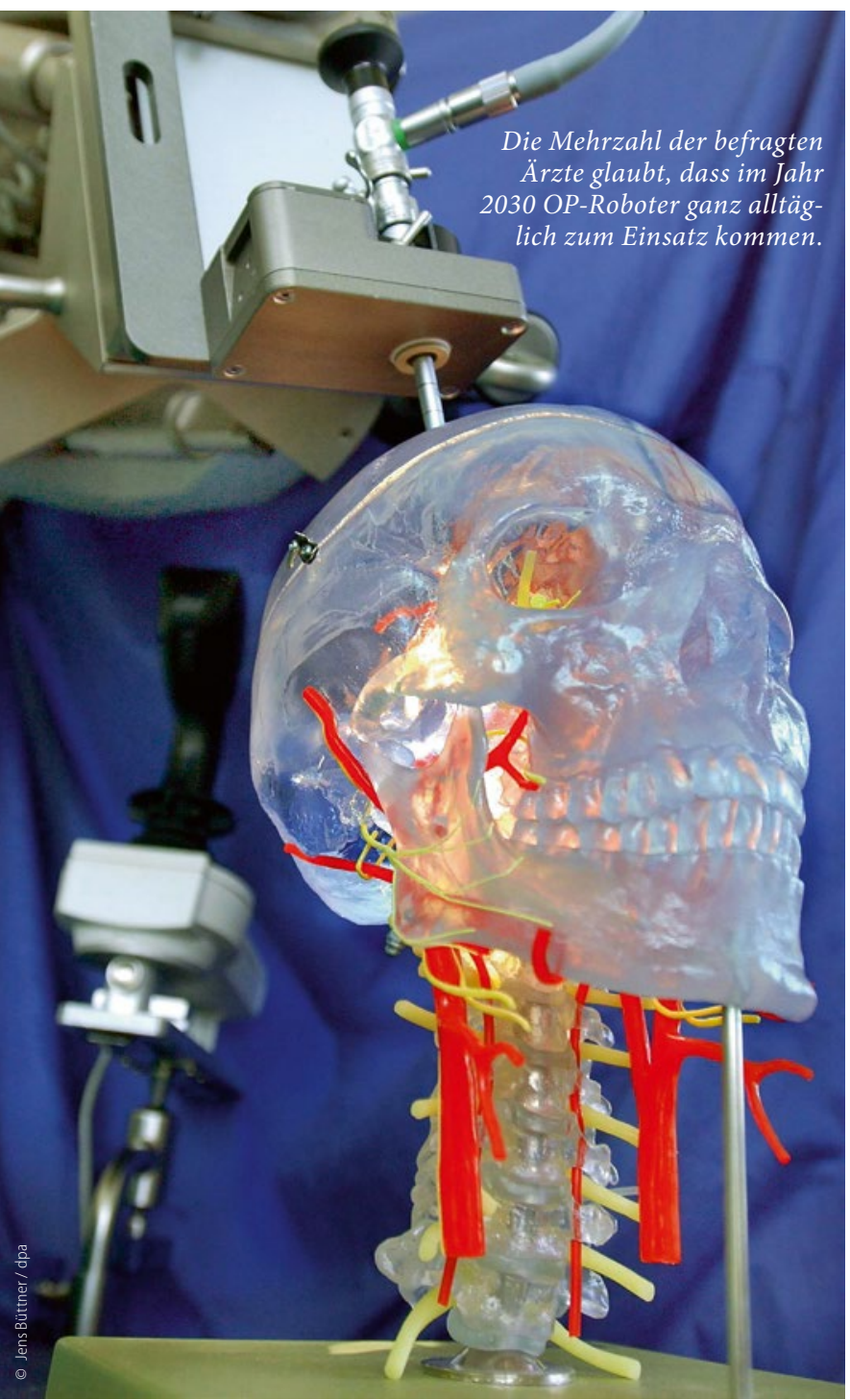

die Versorgung. So das Ergebnis einer gemeinsamen Umfrage des Branchenverbands Bitkom und des Hartmannbundes unter 477 Medizinern aus Praxis und Klinik.

Mehr als jeder zweite Arzt ist demnach der Meinung, dass ihm digitale Anwendungen eine Zeitersparnis bringen. $44 \%$ sagen, dass die neuen Technologien Ärzten bei der Behandlung mehr Möglichkeiten an die Hand geben. Und $39 \%$ glauben, dass dadurch auch in ländlichen Regionen eine flächendeckende Versorgung möglich ist.

„Die Skepsis der vergangenen Jahre ist einer neuen Offenheit gegenüber digitalen Technologien gewichen", sagt Bitkom-Hauptgeschäftsführer Dr. Bernhard Rohleder. Eine Entwicklung, die auch Dr. Klaus Reinhardt, Bundesvorsitzender des Hartmannbundes, wahrnimmt. „Die allgemeine Akzeptanz unter den Ärzten ist wesentlich größer geworden." In der Vergangenheit hätten die Ärzte sich eher auf die Risiken der Digitalisierung fokussiert, aber nun auch erkannt, dass sie an der Technik nicht vorbeikommen. Jetzt gehe es darum, aktiv mitzugestalten. „Wir wollen Taktgeber werden“, so Reinhardt.

In die Zukunft geblickt glauben immerhin $86 \%$ der befragten Ärzte, dass im Jahr 2030 OP-Roboter alltäglich $(47 \%)$ oder zumindest
Noch sind Brief und Fax beliebt

- $37 \%$ der befragten Ärzte kommunizieren überwiegend per Briefpost mit den Krankenkassen, $13 \%$ per Fax und nur $6 \%$ per E-Mail; der Rest gab nichts an oder nutzte nur das Telefon.

- Über ein Viertel setzt auch bei der Kommunikation mit anderen Praxen auf die Post, $16 \%$ nutzen das Fax, $6 \%$ E-Mail

Befragt wurden 477 Ärzte aus Praxis und Klinik.

teilweise zum Einsatz kommen werden. Rund zwei Drittel sind der Meinung, dass künstliche Intelligenz die Ärzte bei Diagnose und Therapie regelmäßig (35\%) oder vereinzelt unterstützen wird. Und $64 \%$ halten den regelhaften Einsatz von unter der Haut implantierten Mikrochips, die die Medikamenteneinnahme und -abgabe steuern, für möglich.

Dass die tatsächliche Vernetzung der Ärzte allerdings noch hinterherhinkt, mag auch an den Hemmnissen liegen, die die Mediziner wahrnehmen. Über zwei Drittel sorgen sich um den Datenschutz und sehen die Gefahr des Missbrauchs von Patientendaten beim Speichern und Übertragen der Daten. Für $63 \%$ stellen Fehler- und Ausfallanfälligkeiten der Medizintechnik ein Risiko dar, 60 \% sorgen sich generell um die IT-Sicherheit medizinischer Einrichtungen. Nach den jüngsten Hackerattacken auch auf britische Kliniken nachzuvollziehen.

Ein gewichtiges Argument dürften überdies die Finanzmittel sein: $43 \%$ der Ärzte sehen schlicht die fehlenden Mittel für die Umsetzung digitaler Anwendungen als eine der Hauptbremsen. Hier stellt Rohleder klar, dass zumindest der Ausbau der Infrastruktur, die ja allen in der Gesundheitsversorgung zugutekomme, von der Allgemeinheit getragen werden müsste und nicht auf den einzelnen Arzt abgewälzt werden dürfe. Die Anschubfinanzierung könne nicht aus den Praxen erfolgen.

RebekkaHöhl 